\title{
O capital social entre adolescentes escolares de um município brasileiro
}

\author{
The social capital among adolescent students of a brazilian \\ municipality
}

Stela Drumond de Menezes Rajão (https://orcid.org/0000-0002-3737-7835) ${ }^{1}$

Patricia Maria Zarzar (http://orcid.org/0000-0002-6952-5767) ${ }^{1}$

Raquel Conceição Ferreira (https://orcid.org/0000-0001-8897-9345) ${ }^{1}$

Efigenia Ferreira e Ferreira (http://orcid.org/0000-0002-0665-211X) ${ }^{1}$
${ }^{1}$ Odontologia Social e Preventiva, Universidade Federal de Minas Gerais (UFMG). Av. Presidente Antônio Carlos 6627, Pampulha. Belo Horizonte MG Brasil steladrum@yahoo.com.br

\begin{abstract}
The article aimed to verify the association of adolescent social capital with living conditions factors and participation in collective activities. In the cross-sectional study, a validated questionnaire (SCQ-AS) was used to measure the social capital of seventh grade students from public and private schools in a Brazilian city. The parents answered a questionnaire on living conditions and children's participation in collective activities. After chi-square test, multiple logistic regression was performed with social capital' domains, dependent variables and independent variables $(p \leq 0.20)$. There was higher frequency of social cohesion at school in adolescents from private schools $(O R=4.08,95 \%$ CI 1.39-11.96) and who have a family income of 1 to 3 minimum wages, a larger network of friends among those with parents living together $O R=2.21,95 \% C I$ 1.28 - 3.78) and higher school/neighborhood confidence in private school students $(O R=2.71,95 \%$ CI 1.16 - 6.32). There was no association with neighborhood social cohesion. Studying in private school and having a family income of 1 to 3 minimum wages can boost social cohesion in school, as well as practice sports. Parents living together can favor larger network of friends.

Keywords Social Capital. Adolescent. Social networking. Social conditions.
\end{abstract}

Resumo O objetivo do artigo foi verificar associação do capital social de adolescentes com os fatores condições de vida e participação em atividades coletivas. No estudo transversal, aplicou-se questionário validado (SCQ-AS) para medir o capital social de escolares do sétimo ano de escolas públicas e privadas de um município brasileiro. Os pais responderam a um questionário sobre condições de vida e participação dos filhos em atividades coletivas. Após teste qui-quadrado, fez-se regressão logística múltipla com os domínios do capital social, variáveis dependentes e variáveis independentes ( $p \leq 0.20)$. Observou-se maior frequência de coesão social na escola, em adolescentes de escolas privadas $(O R=4.08$; IC 95\% 1.39 - 11.96) e com renda familiar de 1 a 3 salários mínimos, maior rede de amigos entre os que tinham pais vivendo juntos $(O R=2.21$; IC 95\% 1.28 - 3.78) e maior frequência de confiança na escola/bairro em escolares de escolas privadas ( $O R=2.71$; IC 95\% 1.16 - 6.32). Não houve associação com coesão social no bairro. Estudar em escola privada e ter renda familiar de 1 a 3 salários mínimos podem impulsionar a coesão social na escola, bem como a pratica de atividade esportiva. Os pais viverem juntos pode favorecer maior rede de amigos.

Palavras-chave Capital social, Adolescente, Rede social, Condições sociais. 


\section{Introdução}

O capital social faz parte da pesquisa contemporânea desde 1985, quando foi definido por Pierre Bourdieu como "a soma dos recursos reais ou potenciais que advém de redes duráveis de relações, mais ou menos institucionalizadas, de conhecimento ou reconhecimento mútuo"1. Segundo o sociólogo francês, a rede social oferece aos seus membros uma "credencial" para acesso a oportunidades impossíveis ou difíceis de serem obtidas individualmente.

$\mathrm{Na}$ área da saúde, o interesse pela pesquisa do capital social foi despertado pelo estudo do cientista político Robert Putnam, na década de 1990, que chamou a atenção para o declínio do capital social nos Estados Unidos e seu impacto negativo no bem-estar geral da sociedade. Sua teoria defendia que "características da organização social como confiança, normas e redes sociais podem melhorar a eficiência da sociedade por facilitar ações coordenadas"”.

A partir desses estudos houve um crescimento progressivo das pesquisas do capital social na saúde, mas ainda hoje há uma vasta discussão sobre o seu conceito e persiste a falta de uma definição consensual para o termo. No entanto, pode-se entendê-lo como um recurso relacionado a redes de relacionamentos, confiança, solidariedade, reciprocidade, normas. O termo capital social apresenta um conceito amplo e difuso porque redes de confiança e solidariedade podem referir-se desde a uma densa rede de organizações e associações civis (tais como ONGs, associações profissionais, de classe, religiosas, de bairros, entidades filantrópicas, cooperativas de produção, grupos em geral) até as conexões sociais mais informais, como relações de amizade ${ }^{3}$.

Diante das possibilidades oferecidas pelo capital social, por meio das redes sociais, é relevante a busca por um conhecimento mais aprofundado desse recurso na adolescência, já que a literatura sobre este tema, nessa fase da vida ainda é escas$\mathrm{sa}^{4}$. Além disso, a adolescência merece atenção especial por ser um período de mudanças, profundamente influenciada pelos relacionamentos. Estudo conduzido na Croácia observou que, o reduzido capital social durante este período vulnerável, pode se constituir em fator de risco para a saúde mental, na idade adulta ${ }^{4}$. O impacto do baixo capital social pode se acumular por todo o curso de vida e é importante reconhecer estes efeitos, especialmente para adolescentes ${ }^{5}$.

Pesquisa com adolescentes brasileiros revelou que as condições socioeconômicas se constituem como fatores de risco para a saúde bucal. Observou-se ainda que, os adolescentes que sempre foram pobres e com maior experiência de pobreza ao longo da vida, apresentaram maior risco em relação às condições de saúde. No entanto, a participação em grupos mostrou-se um fator de proteção ${ }^{6}$. Estudo desenvolvido na Islândia observou que o adolescente é influenciado pelo contexto em que vive. A desigualdade de renda da vizinhança contribui para angústia emocional individual do adolescente, interferindo em sua rede familiar e social ${ }^{7}$.

A importância de mais estudos sobre o capital social dos adolescentes, especificamente sobre a associação de seus indicadores como coesão social, rede de amigos e confiança, com fatores socioeconômicos e participação de atividades em grupos, impulsionou o desenvolvimento desta pesquisa. Pretende-se, neste estudo, avaliar o capital social entre adolescentes escolares e sua associação às condições de vida e nível de participação em atividades coletivas.

\section{Método}

\section{$O$ contexto da pesquisa}

O município do estudo foi emancipado em 1891 e em 1923 recebeu o nome de Nova Lima, o que permanece até a atualidade. Recebeu forte influência da colonização inglesa motivada pela exploração do ouro, fortalecida no início do século 20. Mas hoje, tem como recurso principal a exploração do ferro. Pertence a Região Metropolitana de Belo Horizonte e em 2016 apresentava população estimada de 91.069 habitantes, Índice de Desenvolvimento Humano (IDH) 0,813, renda per capita média de 2,3 salários mínimos e Coeficiente de Gini de 0,4. Com área total de cerca de $430 \mathrm{~m}^{2}$, sua população se concentra na parte central da cidade (origem do município).

A região abriga 11 unidades de preservação ambiental e mais de 800 nascentes e alguns lagos, tendo sido por isto, motivo de uma grande expansão imobiliária ocorrida a partir da década de 80 . Tornou-se um reduto de moradia tranquila para cidadãos que trabalham e vivem em Belo Horizonte, o que motivou o surgimento de condomínios, a maioria fora da área central, habitados por moradores que não vivem no município propriamente dito (a área central). Por este motivo, este estudo tem como foco esta área central, núcleo onde é possível observar as relações entre os adolescentes que vivem integralmente em Nova Lima. 
A região favorece a pratica de atividades físicas e os adolescentes têm a oportunidade de participar de grupos, como no Projeto "Proteger é Preciso", em parceria com uma mineradora ou no "Coletivo Mega-Foco", além de outros grupos de atividades esportivas, artísticas e culturais. Estas ações parecem favorecer a construção de relações sociais e possibilitar uma vida com maior qualidade.

\section{A pesquisa}

Optou-se pelo desenho transversal, utilizando para a coleta de dados o Social Capital Questionnaire for Adolescent Students (SCQ-AS), construído e validado no Brasil, para escolares de 12 anos de idade 8 .

Considerado de boa aplicabilidade e fácil compreensão, apresenta doze questões divididas em quatro domínios: 1. Coesão Social na Escola (os alunos da minha escola ficam juntos, eu sinto que pertenço a esta escola como se ela fosse minha, eu me sinto seguro nesta escola, meus pais se dão bem com meus professores); 2. Rede de Amigos na Escola (os alunos da minha escola se divertem bem uns com os outros, eu confio nos meus amigos da escola, eu posso pedir ajuda aos meus amigos da escola); 3. Coesão Social no Bairro/Vizinhança (eu confio nos vizinhos, eu posso contar com a ajuda dos meus vizinhos); 4. Confiança na Escola e Bairro/ Vizinhança (os professores da minha escola são solidários e nos dão apoio, os meus vizinhos tentariam tirar vantagens de mim, os colegas da escola tentariam tirar vantagens de mim). As três possibilidades de resposta eram iguais para todas as questões: "Concordo"; "Não tenho opinião, não sei" e "Discordo"s.

Para facilitar a coleta de dados, optou-se por aplicar o questionário na própria sala de aula do escolar. O $7^{\circ}$ ano do ensino fundamental foi eleito por concentrar os escolares na faixa etária de interesse do estudo (12 anos). Foram incluídas todas as nove escolas com $7^{\circ}$ ano, no município, localizadas em bairros distintos: 5 estaduais e 4 privadas. Foram excluídas do estudo as escolas privadas (cinco), não inseridas na área central, por contarem com escolares moradores de condomínios, que não convivem no município. $\mathrm{O}$ total de matriculados no sétimo ano das escolas incluídas no estudo foi de 1001 escolares, segundo informações de matrícula no ano de 2017, fornecidas pelas próprias escolas.

Os pais desses escolares também participaram da pesquisa, por meio do preenchimento de um questionário autoaplicável, no qual se procu- rou verificar a renda familiar, situação conjugal dos pais, idade da mãe e trabalho da mãe, além de dados sobre a participação dos seus filhos em atividades coletivas, como esportivas, religiosas e artísticas.

O cálculo amostral foi feito para estimativa de proporção, considerando a significância de 95\%, erro de $5 \%$ e padrão de frequência esperada de $50 \%$, obtendo-se o total de 384 adolescentes. Com uma possível perda de $10 \%$ e deff 1.2 , encontrouse um $n$ de 510 adolescentes. Esse valor foi ajustado por se tratar de um universo finito $(\mathrm{N}=1001)$, chegando-se a um $n$ final de 337 adolescentes.

Foram considerados critérios de inclusão dos escolares: estar regularmente matriculado nas escolas públicas e privadas da área central do município, cursar o $7^{\circ}$ ano do ensino fundamental, não apresentar limitação cognitiva (informação da professora). Os critérios de exclusão foram: não residir no município e estudar em escolas não pertencentes à região central do município.

Um estudo piloto foi desenvolvido com 40 escolares, regularmente matriculados no $7^{\circ}$ ano do ensino fundamental, de uma escola pública estadual de um município próximo ao do estudo, com características sociais semelhantes, tendo como critérios de inclusão estar no $7^{\circ}$ ano do ensino fundamental e não apresentar limitações cognitivas.

Em um primeiro momento a pesquisadora principal compareceu à escola e, com a autorização da direção da escola e da professora, fez a apresentação sobre a pesquisa, aos alunos, em sala de aula. O Termo de Consentimento Livre e Esclarecido (TCLE) foi enviado para os pais, em duas vias, com explicações sobre a pesquisa, solicitando a autorização para participação dos filhos, por meio da assinatura. No segundo encontro, os termos assinados foram recolhidos e os escolares também assinaram o Termo de Assentimento Livre e Esclarecido (TALE), concordando em responder o questionário sobre capital social.

Nessa ocasião, os alunos foram divididos em dois grupos de 20 escolares em salas separadas, para que se definisse a melhor forma de aplicar o instrumento. O primeiro grupo foi orientado a ler e responder o questionário, em silêncio, $\mathrm{e}$ levantar a mão em caso de dúvida, que seria prontamente esclarecida pela pesquisadora. $\mathrm{O}$ segundo grupo recebeu instruções para aguardar a leitura, em voz alta, pela pesquisadora, e em seguida, marcar as opções desejadas. As dúvidas também seriam imediatamente solucionadas pela mesma. A avaliação dos dois métodos permitiu eleger a leitura das questões em voz alta, 
pela pesquisadora, como a melhor forma de se aplicar o questionário, pois possibilitou um maior entendimento pelos alunos, que nesse caso não tiveram dúvidas, além de ter gerado um menor tempo de aplicação.

Após o estudo piloto, a coleta dos dados foi realizada no período de fevereiro a março de 2017 com a autorização da Secretaria de Educação de Minas Gerais e consentimento da diretoria e professores das nove escolas participantes. Os procedimentos de primeiro contato e envio do TCLE para os pais, agora acompanhado do questionário a ser preenchido por eles, foram feitos como no estudo piloto. Foram enviados dois TCLE: um para sua participação no estudo e outro para consentir a participação do(a) filho(a) na pesquisa, cada um em duas vias (uma para os pais e outra para guarda dos pesquisadores).

O questionário preenchido e uma via de cada termo assinado foram recolhidos em um segundo encontro com os escolares, geralmente um dia após a entrega, para se evitar perdas. Nesse segundo encontro, os Termos de Assentimento Livre e Esclarecido (TALE) foram distribuídos para todos os adolescentes que estavam autorizados a participar da pesquisa, assinando a concordância em contribuir para o estudo. Finalmente, os questionários sobre o capital social foram aplicados a esses escolares, também em sala de aula. Todas as questões foram lidas em voz alta pela pesquisadora e foi aguardado que todos os participantes respondessem a cada questão para minimizar viés de compreensão e facilitar o entendimento. As poucas dúvidas que surgiram foram prontamente esclarecidas pela mesma.

A análise do capital social, considerado variável dependente, foi realizada por domínio, buscando-se verificar sua associação com as variáveis independentes: tipo de escola, idade, gênero, renda familiar, trabalho da mãe, idade da mãe, situação conjugal dos pais e participação dos adolescentes em atividades coletivas. Foi atribuído escore 3, 2 ou 1 às respostas "Concordo", "Não tenho opinião/não sei”, "Discordo", respectivamente, nas questões de 1 a 10 do questionário. Apenas nas questões 11 e 12 tiveram seus escores invertidos (perguntas negativas).

A soma dos escores poderia variar de 4 a 12 , de 3 a 9, de 2 a 6 e de 3 a 9 nos domínios 1, 2, 3 e 4 , respectivamente. A partir da soma, cada domínio foi categorizado pelo quartil e classificado em menor, quando o valor da soma dos escores foi menor ou igual ao valor do primeiro quartil e maior, quando esse valor foi superior ao valor do primeiro quartil ${ }^{8}$.
Após a análise descritiva da distribuição da amostra segundo todas as variáveis, realizou-se o teste qui-quadrado para verificar a associação entre cada domínio e as variáveis independentes de interesse. A partir desses resultados, foram selecionadas as que apresentaram o valor $\mathrm{p} \leq 0.20 \mathrm{e}$ realizada regressão logística múltipla.

Este estudo foi aprovado pelo Comitê de Ética em Pesquisa da Universidade Federal de Minas Gerais - COEP, no dia 08 de fevereiro de 2017.

\section{Resultados}

Participaram do estudo, 335 adolescentes, selecionados por conveniência, mediante a apresentação do TCLE assinado pelos pais e o TALE assinado por eles. Entre os pais participantes, 326 entregaram os questionários preenchidos, com algumas questões não respondidas, sendo a variável trabalho da mãe $(6,5 \%)$ e renda familiar $(3,1 \%)$ as duas com maiores perdas.

O capital social total, embora não tenha sido objeto deste estudo, foi calculado e considerado maior na maioria dos adolescentes $(72,7 \%)$, com diferença entre os escolares de escola pública $(64,34 \%)$ e privada $(95,24 \%)$. A média da pontuação do capital social ( 1 a 36 pontos) em escolas públicas foi $26,73 \pm 4,02$ e em escolas privadas foi $31,1 \pm 2,26$. Nos dois tipos de escola foi observada a pontuação máxima (36), mas a pontuação mínima foi de 15 e 25 nas escolas públicas e privadas, respectivamente.

A Tabela 1 mostra a distribuição dos escolares segundo as variáveis de condições de vida e participação em atividades em grupo. Mais de dois terços dos adolescentes da amostra estudavam em escolas públicas. A renda familiar mais frequente nas famílias dos escolares foi de um a três salários mínimos (de $\mathrm{R} \$ 880,00$ até $\mathrm{R} \$ 2.640,00$ ) e a maioria dos pais viviam juntos. A maior parte dos adolescentes, mais de dois terços, participava de alguma atividade coletiva, com destaque para as atividades esportivas em relação às outras atividades.

A Tabela 2 traz a distribuição da amostra de escolares segundo os domínios do capital social. A maior parte dos adolescentes foi considerada de maior capital social, em todos os domínios: coesão social na escola, rede de amigos na escola, coesão social no bairro/vizinhança e confiança na escola e no bairro/vizinhança.

A partir dos resultados da análise binaria, foram selecionadas as variáveis independentes que apresentaram valor de $\mathrm{p} \leq 0,20$. Em relação 
Tabela 1. Distribuição da amostra de escolares adolescentes, segundo condições de vida e participação em atividades em grupo $(\mathrm{n}=335)$

\begin{tabular}{|c|c|c|}
\hline Variável & n (\%) & IC 95\% \\
\hline Tipo de escola & $\mathrm{n}=335$ & \\
\hline Pública & $272(81,2)$ & $76,6-85,0$ \\
\hline Privada & $63(18,8)$ & $14,9-23,4$ \\
\hline Idade & $\mathrm{n}=335$ & \\
\hline 11 ou 12 anos & $255(76,1)$ & $71,2-80,4$ \\
\hline 13 a 16 anos & $80(23,9)$ & $19,6-28,8$ \\
\hline Gênero & $\mathrm{n}=335$ & \\
\hline Feminino & $191(57,1)$ & $51,8-62,4$ \\
\hline Masculino & $144(42,9)$ & $37,6-48,2$ \\
\hline Situação dos pais & $\mathrm{n}=326$ & \\
\hline Vivem juntos & $175(53,7)$ & $48,2-59,1$ \\
\hline Não vivem juntos & $151(46,3)$ & $40,9-51,8$ \\
\hline Idade da mãe & $\mathrm{n}=316$ & \\
\hline Até 37 anos & $162(51,1)$ & $45,6-56,6$ \\
\hline 38 anos ou mais & $154(48,9)$ & $43,4-54,4$ \\
\hline Trabalho da mãe & $\mathrm{n}=305$ & \\
\hline Do lar/Desempregada/Não trabalha & $56(18,4)$ & $14,4-23,1$ \\
\hline Doméstica/Faxineira/Diarista/Babá & $59(19,3)$ & $15,3-24,2$ \\
\hline Profissão de nível fundamental/médio & $112(36,7)$ & $31,5-42,3$ \\
\hline Funcionária pública/Autônoma/Nível superior & $49(16,1)$ & $12,3-20,7$ \\
\hline Outros & $29(9,5)$ & $6,7-13,4$ \\
\hline Renda familiar & $\mathrm{n}=318$ & \\
\hline Nenhuma renda até 1 salário mínimo ( $\$ 880,00)$ & $106(33,3)$ & $28,3-38,7$ \\
\hline De 1 a 3 salários mínimos (até $\mathrm{R} \$ 2640,00$ ) & $149(46,9)$ & $41,4-52,4$ \\
\hline Mais de 3 salários mínimos ( $\mathrm{R} \$ 2640,01$ ou mais) & $63(19,8)$ & $15,8-24,6$ \\
\hline Participação em atividades coletivas & $\mathrm{n}=320$ & \\
\hline Sim & $240(74,8)$ & $69,3-78,9$ \\
\hline Não & $80(25,2)$ & $21,1-30,7$ \\
\hline Participação em atividades esportivas & $\mathrm{n}=320$ & \\
\hline Sim & $159(49,7)$ & $44,2-55,2$ \\
\hline Não & $161(50,3)$ & $44,8-55,8$ \\
\hline Participação em atividades artísticas & $\mathrm{n}=320$ & \\
\hline Sim & $33(10,3)$ & $7,4-14,2$ \\
\hline Não & $287(89,7)$ & $85,8-92,6$ \\
\hline Participação em atividades religiosas & $\mathrm{n}=320$ & \\
\hline Sim & $93(29,1)$ & $24,3-34,3$ \\
\hline Não & $227(70,9)$ & $65,7-75,7$ \\
\hline Hábito de sair com amigos & $\mathrm{n}=320$ & \\
\hline Sim & $67(20,9)$ & $16,8-25,8$ \\
\hline Não & $253(79,1)$ & $74,2-83,2$ \\
\hline Total de atividades relatadas & $\mathrm{n}=318$ & \\
\hline 0 & $80(25,2)$ & $20,7-30,2$ \\
\hline 1 & $140(44,0)$ & $38,6-49,6$ \\
\hline$>1$ & $98(30,8)$ & $25,9-36,1$ \\
\hline
\end{tabular}

ao domínio coesão social na escola, foram selecionadas as variáveis tipo de escola $(\mathrm{p}=0,000)$, gênero ( $\mathrm{p}=0,149)$, situação conjugal dos pais $(p=0,077)$, trabalho da mãe $(p=0,004)$, renda familiar $(\mathrm{p}=0,000)$, participação em atividades esportivas $(\mathrm{p}=0,122)$, participação em atividades artísticas $(\mathrm{p}=0,042)$, total de atividades relatadas $(p=0,003)$ e participação em atividades coletivas $(\mathrm{p}=0,121)$. Em relação ao dominio rede de amigos na escola, foram selecionadas as variáveis tipo de escola $(p=0,001)$, situação conjugal dos pais $(\mathrm{p}=0,037)$, trabalho da mãe $(\mathrm{p}=0,200)$ e ren- 
Tabela 2. Distribuição da amostra de escolares adolescentes, segundo os domínios do capital social $(\mathrm{n}=335), 2017$.

\begin{tabular}{lcc}
\hline $\begin{array}{c}\text { Domínios do Capital } \\
\text { social }\end{array}$ & $\mathbf{n}(\%)$ & IC 95\% \\
\hline $\begin{array}{l}\text { Domínio 1-Coesão } \\
\text { social na escola } \\
\quad \text { Menor }\end{array}$ & $101(30,5)$ & $25,8-35,7$ \\
$\quad$ Maior & $230(69,5) 331$ & $64,3-74,2$ \\
Domínio 2- Rede de & & \\
amigos na escola & & \\
$\quad$ Menor & $94(28,2)$ & $23,6-33,3$ \\
$\quad$ Maior & $239(71,8) 333$ & $66,7-76,4$ \\
$\begin{array}{l}\text { Domínio 3-Coesão } \\
\text { social na vizinhança }\end{array}$ & & \\
$\quad$ Menor & $120(36,3)$ & $31,2-41,6$ \\
$\quad$ Maior & $211(63,7) 331$ & $58,4-68,8$ \\
Domínio 4-Confiança & & \\
na escola/ vizinhança & & \\
$\quad$ Menor & $129(39,7)$ & $34,5-45,1$ \\
$\quad$ Maior & $196(60,3) 325$ & $54,9-65,5$ \\
\hline
\end{tabular}

da familiar $(\mathrm{p}=0,009)$. O domínio coesão social na escola/vizinhança não apresentou associação com nenhuma das variáveis analisadas no teste qui-quadrado. A confiança na escola e no bairro/vizinhança, quarto e último domínio, mostrou relação com as variáveis tipo de escola $(\mathrm{p}=0,000)$, idade $(\mathrm{p}=0,069)$, situação conjugal dos pais $(p=0,104)$, trabalho da mãe $(p=0,050)$, renda familiar $(\mathrm{p}=0,019)$, participação em atividades esportivas $(\mathrm{p}=0.076)$, hábito de sair com amigos $(p=0.015)$, total de atividades relatadas $(\mathrm{p}=0,148)$ e participação em atividades coletivas $(\mathrm{p}=0,086)$. Os dados da regressão múltipla se encontram descritos na Tabela 3.

A Figura 1 mostra a figura representativa de um modelo explicativo para este estudo, apresentando uma síntese das relações estabelecidas entre as variáveis.

\section{Discussão}

Considerando os domínios do capital social, medidos pelo Social Capital Questionnaire for Adolescent Students (SCQ-AS), observou-se neste estudo a presença de alto capital social entre adolescentes, tanto considerando o total geral (72,7\%), como por domínios: Rede de amigos na escola (71,8\%), Coesão social na escola (69,5\%), Coesão social na vizinhança $(63,7 \%)$ e Confiança na escola/ vizinhança $(60,3 \%)$.
O maior capital social está geralmente associado a melhores condições socioeconômicas e de saúde ${ }^{9-12}$. Como nosso estudo foi realizado em um município de alto IDH, ou seja, com bons indicadores de renda, educação e longevidade, há um contexto que pode ter contribuído para o maior nível de capital social total e de seus domínios. No entanto, os melhores resultados foram observados em escolas privadas, onde estes indicadores são mais frequentes.

O primeiro domínio do capital social analisado, coesão social na escola, se refere às relações próximas com os colegas, entre a família (pais) e a escola e ao grau de pertencimento àquele espaço. Este se mostrou associado para adolescentes que vivem em famílias de renda média (OR 2,45; 1,39-11,96), estudando em escola privada (OR $4,08 ; 1,39-11,96)$ e ainda entre as mulheres e entre os participantes de atividades esportivas.

Os escolares de escolas privadas, de um modo geral, pertencem a famílias com maior renda. Geralmente, esses adolescentes com melhores condições socioeconômicas apresentam maior frequência de coesão social, enquanto que os de menor renda familiar relatam não se sentirem seguros no ambiente escolar e reportam conexões mais pobres com suas escolas, fatores que indicam menor coesão social. A diferença de padrões socioeconômicos também influencia o apoio dos pais à escola e a percepção dos adolescentes sobre suas relações com a escola em termos de regras institucionais e bullying ${ }^{13}$.

A categoria renda que apresentou associação com a coesão social na escola foi a intermediária (um a três salários mínimos), o que sugere a possível influência de outros fatores nos níveis de coesão social na escola, por exemplo a importância da coesão familiar e os relacionamentos com amigos próximos, que também estão associados a uma maior percepção de segurança na escola pelos adolescentes ${ }^{14}$.

A menor frequência de coesão social na escola foi encontrada entre os adolescentes do sexo masculino e que não praticavam atividades esportivas. Essas associações estão de acordo com estudos que indicam uma maior tendência das adolescentes do gênero feminino a relatarem maiores níveis de capital social em relação aos do gênero masculino ${ }^{4}$. Além disso, existem outros dados sobre a coesão social, especificamente, que sugerem fortes associações de gênero quanto à forma como os adolescentes se relacionam com seus pais em termos da escola e mostram uma maior preocupação das meninas com esse relacionamento $^{13}$. 
Tabela 3. Regressão logística múltipla dos fatores associados aos domínios do Capital Social de escolares adolescentes, 2017.

\begin{tabular}{|c|c|c|c|}
\hline Variáveis independentes & Odds Ratio & p & IC $95 \%$ \\
\hline \multicolumn{4}{|c|}{ Coesão Social na Escola $* *$} \\
\hline \multicolumn{4}{|l|}{ Tipo de escola } \\
\hline Pública & 1 & & \\
\hline Privada & 4,08 & $0,010^{*}$ & $1,39-11,96$ \\
\hline \multicolumn{4}{|l|}{ Renda familiar } \\
\hline Nenhuma renda até 1 salário mínimo (até $\mathrm{R} \$ 880,00$ ) & 1 & & \\
\hline De 1 a 3 salários mínimos (até $\mathrm{R} \$ 2640,00$ ) & 2,45 & $0,002^{*}$ & $1,39-4,28$ \\
\hline Mais de 3 salários mínimos ( $\mathrm{R} \$ 2640,01$ ou mais) & 1,74 & 0,252 & $0,67-4,46$ \\
\hline \multicolumn{4}{|l|}{ Gênero } \\
\hline Feminino & 1 & & \\
\hline Masculino & 0,41 & $0,028^{*}$ & $0,18-0,90$ \\
\hline \multicolumn{4}{|l|}{ Participação em atividades esportivas } \\
\hline Sim & 1 & & \\
\hline Não & 0,43 & $0,035^{\star}$ & $0,20-0,94$ \\
\hline \multicolumn{4}{|c|}{ Rede de Amigos na Escola ${ }^{* * *}$} \\
\hline \multicolumn{4}{|l|}{ Situação dos pais } \\
\hline Não vivem juntos & 1 & & \\
\hline Vivem juntos & 2,21 & $0,004^{*}$ & $1,28-3,78$ \\
\hline \multicolumn{4}{|c|}{ Confiança na Escola/Vizinhança ${ }^{\star * * *}$} \\
\hline \multicolumn{4}{|l|}{ Tipo de escola } \\
\hline Pública & 1 & & \\
\hline Privada & 2,71 & $0,021^{*}$ & $1,16-6,32$ \\
\hline
\end{tabular}

\section{Domínios}

do capital social

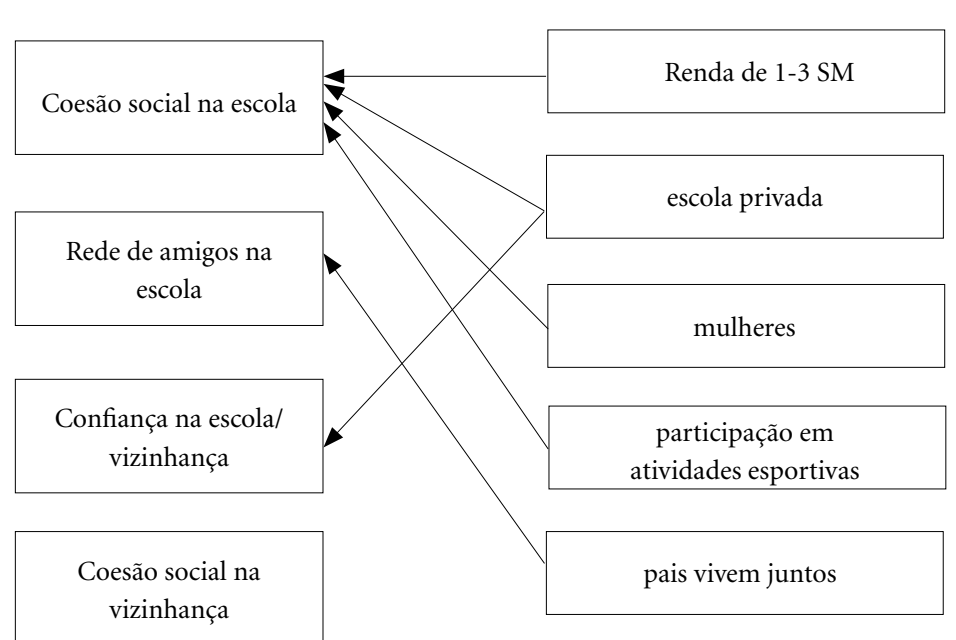

\section{Condições de vida}

Renda de 1-3 SM . 
Neste grupo de escolares, entre as atividades coletivas, somente as esportivas influenciaram o capital social, no domínio coesão social na escola. Uma pesquisa recente sobre coesão social e atividades físicas nas escolas também mostrou que esportes em equipe parecem ser mais vantajosos para o desenvolvimento da diversão e da coesão, fatores que influenciam positivamente os resultados de saúde ${ }^{15}$. Esses dados corroboram os resultados do nosso estudo.

A análise do domínio rede de amigos na escola mostrou que a maioria dos adolescentes deste estudo apresentou maior rede de amigos $(71,8 \%)$ e sua maior frequência foi encontrada entre os adolescentes que pertenciam a famílias em que os pais viviam juntos (OR 2,21; 1,28-3,78).

Essa foi a única associação verificada em relação à rede de amigos na escola e sugere a influência dos laços familiares nas relações dos adolescentes com os amigos, principalmente quanto à confiança e solidariedade, abordadas nas questões desse domínio. Para pessoas jovens, a família é importante por estar lá nas horas que eles mais precisam e os familiares são sempre considerados como um recurso crucial de apoio ${ }^{4}$. Pesquisas já mostraram que não viver com ambos os pais biológicos aumenta a chance de transtorno afetivo em adolescentes ${ }^{16} \mathrm{e}$ este pode refletir uma dificuldade em se relacionar e interferir negativamente na construção de uma rede de amizades pelos adolescentes. Os estudos de Coleman já levantavam evidências sobre o impacto positivo das famílias monoparentais na deserção escolar, que também pode ser um fator que dificulta a criação de laços de amizade entre os estudantes ${ }^{17}$.

A confiança dos adolescentes na escola e no bairro/vizinhança, outro domínio do capital social, foi mensurada em nosso estudo por meio de questões sobre solidariedade, apoio dos professores e confiança dos adolescentes em seus colegas de escola e vizinhos e foi considerada maior na maioria dos adolescentes (60,3\%). A análise desses dados mostrou uma maior frequência de confiança na escola e no bairro/vizinhança entre adolescentes de escolas privadas (OR 2,71; 1,16$6,32)$.

Considerando as melhores condições socioeconômicas dos adolescentes de escolas privadas em relação aos de escolas públicas, de modo geral, admite-se que aqueles tendem a residir em bairros mais favorecidos. Assim, esse resultado se apoia no modelo das normas e eficácia coletiva, por Leventhal e Brooks-Gunn ${ }^{18}$, em que as desvantagens estruturais do bairro influenciam negativamente as normas sociais da vizinhança. Em contrapartida, bairros mais favorecidos tendem a ter maior boa vontade da vizinhança em intervir pelo bem comum e isso repercute positivamente nos resultados para crianças e adolescentes ${ }^{19}$. Esse modelo pode explicar nossos resultados na medida em que os melhores níveis de solidariedade entre vizinhos e colegas de escola e, portanto, maior confiança na escola e no bairro/vizinhança, foram encontrados em adolescentes de escolas privadas, que tendem a residir em bairros mais favorecidos.

A coesão social na vizinhança, apesar de ter sido considerada alta na maioria dos adolescentes $(63,7 \%)$ não apresentou associação com nenhuma das variáveis independentes analisadas. Esse resultado pode ser explicado pelas particularidades do capital social dos adolescentes em relação ao capital social de adultos, pois "comunidades de pessoas jovens constituem mais frequentemente uma comunidade virtual de amigos em torno da escola, centro da cidade e rua, casas de amigos e parentes, ao invés de fortemente ligada a uma localização geográfica identificável facilmente" 20 .

Uma outra possibilidade para o resultado da coesão social na vizinhança em nosso estudo encontra apoio em outro modelo de Leventhal e Brooks-Gunn ${ }^{18}$ chamado modelo dos recursos institucionais, em que a qualidade, acessibilidade e disponibilidade dos recursos institucionais podem explicar a relação entre as características da vizinhança e os resultados em crianças e adolescentes. Esse modelo sugere que a quantidade e qualidade de recursos que influenciam a vida dos jovens (por exemplo, atividades de lazer, educação, saúde, facilidades de cuidado) tendem a ser mais baixas em vizinhanças desfavorecidas (baixo status socioeconômico, elevada diversidade étnica, alta instabilidade residencial) ${ }^{19}$.

Segundo Kawachi ${ }^{21}$, estudos que tentaram relacionar saúde e capital social demonstraram que estas associações são facilmente observadas em países com expressivos níveis de desigualdade. O mesmo fenômeno é verificado com relação à associação entre saúde e renda. Menor capital social e menor nível de renda trazem como consequência redes de segurança inadequadas e comportamentos cooperativos escassos, panorama também observado no presente estudo.

Neste caso, o acumulo de capital social aumenta de importância, já que, para se chegar a determinados objetivos, como a prática da promoção de saúde, o grupo deveria ser capaz de acessar recursos fora do seu meio social. Reforçando o capital social, sobretudo entre os esco- 
lares da rede pública, seria possível se ter "acesso a oportunidades impossíveis ou difíceis de serem obtidas individualmente" ${ }^{\text {, }}$ atraindo a credibilidade, que pode resultar em legitimidade, e um contrato social mais forte $\mathrm{e}^{22}$.

Considerando que, entre os fatores associados estudados, a atividade física é o único que pode ser utilizado de imediato, e considerando sua associação com a coesão na escola, este parece ser um bom caminho para um primeiro passo. Um exercício de empoderamento entre os escolares.

Reconhecemos que o estudo tem limitações, inicialmente por ser transversal, que enfraquece as associações e não permite inferir a determinação. Além disto, a menor amostra de escolares de escolas privadas em relação aos de escolas públicas, também pode ter sido uma limitação. No entanto a distribuição de escolares matriculados em escolas públicas e privadas do município do estudo (cerca de $20 \%$ em escolas privadas e $80 \%$ em escolas públicas) representa o quadro nacional em escolas de ensino fundamental e médio no Brasil.

\section{Considerações finais}

Observando o modelo construído (Figura 1), nota-se que, apesar do município apresentar o melhor IDH do estado, observou-se a desigualdade entre os escolares com prejuízo para frequentadores de escolas públicas. A escola privada está associada à maior coesão social na escola e maior confiança na escola/vizinhança.
A escola pública na maioria das vezes não é escolha, ao contrário da escola privada. A renda familiar é determinante desta opção e observando a renda familiar dos adolescentes deste estudo, a menor renda foi associada também a menor coesão social na escola. Deste modo, desigualdade e capital social são complementares e não concorrentes $^{21}$, mesmo se tratando de questões empíricas.

A desigualdade de renda em uma comunidade pode gerar angústia emocional em seus adolescentes, interferindo em suas redes familiares e relações sociais ${ }^{7}$. O estabelecimento de relações sociais se constituem na fonte do capital social e o menor capital social, sobretudo considerando o período vulnerável da adolescência, pode contribuir para uma trajetória de vida com riscos para saúde física e mental ${ }^{5}$.

Este é o trabalho que se vislumbra pela frente. Entende-se o capital social como um recurso, para melhoria das condições de vida dos adolescentes, sobretudo os que ainda não recebem a atenção necessária das políticas públicas. Os resultados sugerem que é preciso olhar além dos problemas individuais dos adolescentes $\mathrm{e}$ enxergar seus relacionamentos e o contexto em que vivem como uma forma de transformar sua vulnerabilidade em benefícios. O município do estudo tem grande potencial para explorar o capital social dos adolescentes, considerado maior em aproximadamente, dois terços da amostra. Sugerimos novos estudos para uma melhor compreensão dos nossos achados.

\section{Colaboradores}

Todos os autores participaram da concepção do estudo, construção do método, análise dos dados e elaboração do artigo. A coleta de dados foi realizada exclusivamente por SD Menezes. 


\section{Referências}

1. Bourdieu P. The forms of Capital. In: Richardson JG, editors. Handbook of theory and research for the sociology of education. New York: Greenwood; 1985. p. 241-258.

2. Putnam R. Making Democracy Work: Civic Traditions in Modern Italy. Princeton: Princeton University Press; 1993.

3. Fernandes ASA. O capital social e a análise institucional e de políticas públicas. Rev Adm Publica 2002;36(3):375-398.

4. Novak D, Suzuki E, Kawachi I. Are family, neighbourhood and school social capital associated with higher self-rated health among Croatian high school students? A population-based study. BMJ Open 2015;5(6):e007184.

5. Jonsson F, Hammarström A, Gustafsson PE. Social capital across the life course and functional somatic symptoms in mid-adulthood. Scand J Public Health 2014;42(7):581-588.

6. Teixeira AKM, Roncalli AG, Noro LRA. Iniquidades na assistência odontológica ao longo do curso de vida de jovens: um estudo de coorte. Ciên Saude Colet 2018;23(1):249-258

7. Vilhjalmsdottir A, Gardarsdottir RB, Bernburg JG, Sigfusdottir ID. Neighborhood income inequality, social capital and emotional distress among adolescents: A population-based study. J. Adolesc 2016;51:92-102.

8. Paiva PCP, Paiva HN, Filho PMO, Lamounier JA, Ferreira EF, Ferreira RC, Kawachi I, Zarzar PM. Development and validation of a social capital questionnaire for adolescent students (SCQ-AS). PloS one 2014;9(8):e103785.

9. Islam MK, Merlo J, Kawachi I, Lindström M, Gerdtham UG. Social capital and health: Does egalitarianism matter? A literature review. Int J Equity Health 2006;5(1):3.

10. Petrou S, Kupek E. Social capital and its relationship with measures of health status: evidence from the Health Survey for England 2003. Health Econ 2008;17(1):127-143.

11. Fujisawa Y, Hamano T, Takegawa S. Social capital and perceived health in Japan: an ecological and multilevel analysis. Soc Sci Med 2009;69(4):500-505.

12. Hurtado D, Kawachi I, Sudarsky J. Social capital and self-rated health in Colombia: the good, the bad and the ugly. Soc Sci Med 2011;72(4):584-590.

13. Due P, Lynch J, Holstein B, Modvig J. Socioeconomic health inequalities among a nationally representative sample of Danish adolescents: the role of different types of social relations. J Epidemiol Community Health 2003;57(9):692-698.

14. Hong JS, Merrin GJ, Crosby S, Jozefowicz DMH, Lee, $\mathrm{JM}$, Allen-Meares P. Individual and contextual factors associated with immigrant youth feeling unsafe in school: a social-ecological analysis. J Immigr Minor Health 2016;18(5):996-1006.
15. Elbe AM, Wikman JM, Zheng M, Larsen MN, Nielsen G, Krustrup P. The importance of cohesion and enjoyment for the fitness improvement of 8-10-yearold children participating in a team and individual sport school-based physical activity intervention. Eur J Sport Sci 2017;17(3):343-350.

16. Cuffe SP, McKeown RE, Addy CL, Garrison CZ. Family and psychosocial risk factors in a longitudinal epidemiological study of adolescents. J Am Acad Child Adolesc Psychiatry 2005;44(2):121-129.

17. Higgins SS. O capital social como infra-estrutura de iniciativas produtivas: estudo de caso de um projeto agroindustrial na Colômbia [tese]. Santa Catarina, França: Universidade Federal de Santa Catarina, Paris-Dauphine; 2008.

18. Leventhal T, Brooks-Gunn J. Changing neighborhoods: Understanding how children may be affected in the coming century. Advances in Life Course Research 2001;6:263-301.

19. Vyncke V, De Clercq B, Stevens V, Costongs C, Barbareschi G, Jónsson SH, Curvo SD, Kebza V, Currie C, Maes L. Does neighbourhood social capital aid in levelling the social gradient in the health and well-being of children and adolescents? A literature review. BMC Public Health 2013;13(1):65.

20. Morrow VM. 'Dirty looks' and 'trampy places' in young people's accounts of community and neighbourhood: Implications for health inequalities. Crit Public Health 2000;10(2):141-152.

21. Kawachi I. Commentary: Social capital and health - making the connections one step at the time. Int J Epidemiol [Internet]. 2006 Aug [acessado 6 Mar 2013];35(4):989-993. Disponível em: http://ije.oxford journals.org/content/35/4/989.full.pdf+html

22. Baquero M. Construindo uma outra sociedade: o capital social na estruturação de uma cultura política participativa no Brasil. Rev Sociol Polit 2003;21:83108.

Artigo apresentado em 04/06/2017 Aprovado em 06/04/2018

Versão final apresentada em 08/04/2018 